\title{
20 ans au Comité central: un bilan
}

\section{Yves Guisan}

Ancien vice-président FMH, ancien conseiller national
20 ans de politique professionnelle, 14 de politique tout court, quel bilan en tirer? Mitigé ou plus exactement partagé entre les satisfactions incontestables que permettent ces fonctions, et par ailleurs un combat permanent, incessant, harassant dans le domaine de la santé, où les succès sont caractérisés bien davantage par un retard des échéances que par un véritable renouveau de la réflexion. Pourtant les dernières perspectives, fortement dépendantes des résultats de dimanche, laissent ouvertes quelques changements positifs.

\section{0 ans de politique professionnelle, 14 de politique tout court, quel bilan en tirer?}

Correspondance:

Dr Yves Guisan

Le Moulin

CH-1660 L'Etivaz

yvesguisan@sapphirenet.gi
Mon activité à la FMH a été marquée d'entrée par les tribulations de l'ancienne Loi sur l'assurance-maladie et accidents LAMA, la transitoire Loi sur l'assurance-maladie et maternité LAMM et maintenant la Loi sur l'assurance-maladie LAMal. J'ai aussi été confronté à trois présidents et un changement très significatif de préoccupations, surtout de conception du rôle du médecin dans le système de santé.

Dans les années 80, la LAMA a été menée à une crise structurelle majeure par abus gravissime de la sélection des risques, les caisses se battant d'autant plus furieusement pour les patients jeunes et en bonne santé que les primes étaient établies en fonction de l'âge et des risques dans le cadre d'une assurance non obligatoire. Dans ce contexte, les personnes âgées n'arrivaient plus à changer de caisse et faisaient face à des primes exorbitantes. Il en résultait aussi une charge de plus en plus lourde pour la Confédération et les cantons qui payaient des contributions individuelles, par enfant, par femme, et par homme, qui plus est, indexées en fonction des coûts de la santé.

Diverses mesures ont été prises pour tenter de faire face à cette crise que l'on peut qualifier d'existentielle. La Confédération a d'abord décidé de bloquer sa contribution à 2 milliards et de ne plus indexer ses contributions sur les coûts. Ensuite elle a tenté un programme d'urgence par le biais de la LAMM, qui introduisait toutes une série de mesures drastiques: la franchise à choix avec une participation maximale de 750 francs par cas, la quote-part ambulatoire à 20\%, 10\% pour les enfants, la participation des patients pour les frais de pension et de traitement en milieu hospitalier, et enfin le médecin-conseil obligatoire pour chaque caisse, ce qui n'était pas le cas à l'époque. La rétribution des médecins et de manière générale des professions paramédicales était soumise à un tour de vis sous forme d'une méthodologie unique pour toute la Suisse dont le Conseil fédéral fixerait les principes le cas échéant. Bref, tout deviendrait rigoureusement contrôlé. Cette LAMM adoptée en mars 1987 par le Parlement a heureusement été rejetée lors d'un referendum voté le 6 décembre 1987. Mais elle a indiscutablement semé quelques jalons pour la suite. C'est la semaine qui a suivi que j'ai été élu en compagnie de Max Giger au Comité central.

Nous avons d'emblée vécu une période très tumultueuse. Une initiative dite des «caissesmaladie» tente de proposer une autre solution de nature à maîtriser les coûts, mais formulée de manière très générale. Elle sera également rejetée par le peuple. Suivront le mandat donné par le Conseil fédéral à quatre experts afin de dégager quelques propositions concrètes. Parallèlement des arrêtés fédéraux urgents sont promulgués pour geler les tarifs, les subventions, et les primes.

Nous vivions à l'époque un comité central très discipliné, très polissé et très formaliste. Le président, le Dr Hans-Ruedi Sahli, était très respectueux de chacun avec un style un peu «vieille France». Dans ce contexte de révision de la LAMA, notre principale préoccupation était le maintien de notre indépendance vis-à-vis des caisses et de la liberté thérapeutique. Les problèmes d'assurance pouvaient certes concerner la relation financière avec l'assuré. Mais nous entendions conserver notre indépendance et notre liberté de conclure ou non des conventions cadre avec les assureurs de façon aussi peu réglementée que possible. Quant à la liberté thérapeutique, il s'agissait là et il s'agit encore d'un principe sacrosaint intouchable. Notre vision de la médecine restait alors très individualiste, limitée à la relation médecin-malade individuelle, mais sans l'intégrer dans un système de santé ou dans une relation de société plus générale. Ce sont les opi- 
nions que le président Sahli et notre ancien secrétaire général François-Xavier Deschenaux ont défendues dans le cadre de la Commission extraparlementaire Otto Schoch, conseiller aux Etats d'Appenzell Rhodes-Extérieures. Cette commission était appelée par le Conseil fédéral à formuler une proposition de révision de la LAMA en s'inspirant de quelques principes de base qu'il avait fixé.

C'est ainsi que nous avons été étroitement mêlés à l'élaboration du rapport d'experts publié le 2 novembre 1990 et qui a précédé d'une année le message du Conseil fédéral concernant la LAMal publié le 6 novembre 1991. Nous sommes intervenus pendant les débats parlementaires d'alors, mais en respectant les règles de déférence vis-à-vis des parlementaires qui prévalaient alors. La LAMal a été finalement soutenue sans enthousiasme et même du bout des lèvres par la FMH. Nous nous sommes d'ailleurs abstenus de toute campagne visible lors de la votation du 4 décembre 1994, nos rangs étant très partagés. C'est d'ailleurs de justesse que la loi qui nous gouverne à présent a été acceptée par le peuple puisque ce fut par 1021175 voix contre 950060, soit une courte majorité de 71115 voix, en d'autre terme $51,8 \%$ de oui.

La FMH s'est alors attelée à l'un des paramètres qui avaient pour nous une signification essentielle: la mise en œuvre de l'art. 43 al. 5 soit les «tarifs à la prestation doivent se fonder sur une structure tarifaire uniforme fixée par convention sur le plan suisse. Si les partenaires tarifaires ne peuvent s'entendre sur une structure tarifaire uniforme, le Conseil fédéral la fixe.» Les travaux ont alors été poursuivis dans la foulée de la révision en cours du tarif «accidents» sous le sigle en allemand de GRAT (Gesamt-Revision des ArztTarifes). Cette révision avait été décidée d'un commun accord et dans une relative bonne entente avec la CTM/MTK en automne 1987.

\section{C'est d'ailleurs de justesse que la LAMal présente a été acceptée par le peuple à une courte majorité de 71115 voix, en d'autre terme $51,8 \%$ de oui}

J'ai été incorporé dans ce groupe de négociation dès mon entrée au CC en 1987, soit dès la mise en œuvre des négociations de principes. Il s'agissait en effet d'établir une base concrète à la notion «d'économie d'entreprise» qui devait permettre de rétablir l'équilibre entre les différentes spécialités et de calculer à l'avenir de nouvelles prestations sans difficultés. Il s'agissait aussi de définir le cadre normatif d'un cabinet médical. Cela a débouché non sans peine autour de 1994 sur les règles de base permettant d'élaborer un nouveau tarif. C'est alors que les caisses maladie se sont jointes à ce groupe de travail à la condition de reprendre sans remise en question les travaux dans leur état. Ce ne fut malheureusement pas le cas et l'apparition $\mathrm{du}$ «Concordat», puis de santésuisse dans le groupe de négociation a considérablement compliqué la tâche. Quoi qu'il en soit et je ne m'appesantirais pas ici sur les détails de cette longue négociation, celle-ci a rapidement quitté les principes établis pour prendre une tournure plus exclusivement technique, et disons le franchement celle d'une négociation de bazar ...

Nous avions également changé de président en 1994. Le nouveau président Hans Heinrich Brunner, aux orientations plus économiques et plus techniques, s'est trouvé très à l'aise dans ce nouveau défi qui est devenu «sa chose» au point que le CC a progressivement été quasiment exclu de tout contrôle des opérations. La qualité de membre quasiment obligatoire de la FMH était menacée à court/moyen terme par l'instauration de diplômes d'Etat pour la formation postgraduée. Par définition ceux-ci doivent être accessibles à tous y compris les non-membres. Dès lors la qualité de membre devait se justifier par des avantages palpables allant au-delà de la formation professionnelle et de l'activité syndicale. L'attractivité de l'association professionnelle devait se voir considérablement renforcée en se basant essentiellement sur des avantages économiques, et seulement accessoirement sur des questions d'intérêt professionnel. Il s'est alors agi de la mise en valeur de FMH Services, de la création de HIN, puis de Bluecare, d'un soutien à AESA, entreprise genevoise de distribution de matériel médical, et par la suite des implications du nouveau tarif TARMED au niveau de la gestion des données. Cette liste n'est pas exhaustive.

Ces opérations n'ont manifestement pas été un succès et ont abouti à des tensions financières extrêmes dans un contexte d'autoritarisme et de désinformation. Vous avez procédé à leur assainissement au cours de cette législature. Mais surtout cette vision technique et économique enrobée dans un discours fallacieux aux apparences très cultivées a passablement manqué de conceptions plus fondamentales du rôle des médecins et de la profession dans le système de santé. Les préoccupations plus exclusivement liées aux rapports de force entre partenaires l'ont manifestement emporté. Cela nous a privés de réponse face aux propositions plus fondamentales et tenaces de la liberté de contracter et du tout à la concurrence économique. 
Ce manque de visions politiques et sociales de la FMH n'a fait que renforcer les tendances excentriques qui l'animent avec la constitution de divers groupements d'intérêts particuliers. Il y a pourtant effectivement d'autres moyens de contrôler les coûts de la santé tout en respectant la mission sociale et de société essentielle du système de santé et tout en restant aussi vigilants quant à nos intérêts professionnels. Nommé viceprésident de la FMH tout d'abord par le CC selon les anciens statuts, puis par vos soins par la suite, il s'est avéré extrêmement difficile pour moi de lancer la discussion sur ces questions pourtant essentielles avec le président. Par ailleurs nous n'étions que quelques-uns à le questionner à ce sujet au sein du comité central pour être soit rapidement remis en place, soit minorisés.

De toute évidence, les temps ont changé. La qualité de médecin ne suffit pas pour obtenir raison simplement en clamant son opposition. Les prérogatives corporatistes d'une fois sont maintenant à juste titre taxées d'arrogance. Il faut être capable de formuler des propositions alternatives et d'argumenter. Dans ce domaine jusqu'à récemment, nous avons été certainement déficients.

C'est ainsi que je me suis trouvé bien seul comme conseiller national dès 1995 . En matière de santé je me suis considéré surtout comme représentant de la FMH. Les propositions multiples que j'ai faites au président $\mathrm{HHB}$ ont dans la règle été acceptées de sa part, non sans que des propos totalement discordants soient proférés par ailleurs dans nos propres rangs et groupements d'intérêts. Ces voix multiples n'ont pas contribué à la crédibilité de mes propositions sur le plan politique.

\section{La qualité de médecin ne suffit pas pour obtenir raison simplement en clamant son opposition}

De toute évidence la LAMal, sans être remise en question quant à ses principes, pose de gros problèmes d'application, qu'il s'agisse de la répartition des compétences entre cantons et Confédération, de la charge à la limite du supportable que représente la solidarité pour la classe moyenne avec des systèmes de subventions cantonaux extrêmement disparates, ou d'un fonds de compensation insuffisant pour exclure la sélection des risques et placer la concurrence entre assu- reurs sur le plan strict de l'efficience et de la qualité. Le financement et la planification hospitaliers ont été partiellement résolus à satisfaction par la décision du Parlement de décembre dernier. Au centre de toutes ces préoccupations se trouve la maîtrise des coûts avec la proposition maintenant devenue récurrente de la liberté de contracter. Ces différentes questions sont apparues toujours et encore au cours des trois révisions de la LAMal que nous avons connues depuis 1999.

Jusqu'à présent nous avons échappé à cette fameuse liberté de contracter qui serait la solution miracle. Mais il s'en est souvent fallu de peu. Une première tentative par le biais d'une initiative parlementaire du conseiller national Hansueli Raggenbass, PDC (TG), directeur de la Sanitas, lancée parallèlement à la première révision de la LAMal en 1999, a tourné court devant le Conseil des Etats.

Par contre la deuxième révision, certes élaborée encore sous l'égide de Mme la conseillère fédérale Ruth Dreifuss a failli mal tourner. Elle prévoyait non seulement la liberté de contracter pour les spécialistes, mais encore divers ajustements pour alléger les familles et surtout de nouvelles règles de financement hospitalier. Je suis l'auteur de son rejet définitif et j'en suis fier. J'ai en effet proposé en décembre 2003 de refuser les propositions de la commission de conciliation, précisément en raison de la mise en place de la liberté de contracter, qui si elle était acceptée ferait l'objet d'un referendum auquel la FMH s'était dument préparée. Le PDC a suivi estimant l'allégement pour les familles avec enfants insuffisant - il souhaitait la libération des primes à partir du troisième enfant - et le PS en a fait de même parce que cette nouvelle loi aurait instauré une libéralisation à l'américaine sans garantie de la qualité ni de la maîtrise des coûts avec en plus un conflit programmé cantons/ Confédération en matière de planification. Le Conseil national a voté le rejet de ce projet par 71 voix contre 66. Cela nous a évité de lancer le referendum que la FMH avait décidé.

On pouvait penser la liberté de contracter enterrée pour un certain temps tout au moins. Cela préjugeait singulièrement de la volonté du conseiller fédéral Pascal Couchepin en charge du département depuis le $1^{\text {er }}$ janvier 2003, et de celle du Conseil fédéral. Après une procédure de consultation orale accélérée voire bâclée, la loi rejetée par le Parlement a été reprise, mais morcelée en plusieurs objets séparés afin d'éviter le rejet global que ce soit par le Parlement ou lors d'un referendum éventuel. Seuls deux objets ont été réglés jusqu'à présent, le premier de nature organisationnelle comportant pour l'essentiel la 
carte de santé, et le deuxième le financement hospitalier. Les autres sont encore en suspens. Le Conseil des Etats, désigné premier Conseil dans l'idée sans doute que cela accélérerait les choses et déboucherait rapidement sur un projet bien ficelé, s'est penché en profondeur sur ces différents volets pour ne pas répéter l'expérience désastreuse du 17 décembre 2003.

\section{Jusqu'à présent nous avons échappé à cette fameuse liberté de contracter qui serait la solution miracle}

Le fonds de compensation est agendé pour la session d'été qui commencera début juin 2008. Il verra une amélioration, incomplète puisqu'elle comporte désormais l'hospitalisation de la dernière année, mais pas de critères de morbidité. La liberté de contracter suivra cet automne et l'hiver prochain. Elle est fortement liée à la prolongation ou non de la clause du besoin, sans que l'on sache d'ailleurs vraiment comment. Diverses solutions partielles peu satisfaisantes continuent à être évoquées, même si plus récemment les positions alternatives de la FMH sont en passe de devenir plus convaincantes. Et enfin, cerise sur le gâteau, le contreprojet à l'initiative UDC «Pour une baisse des primes d'assurance-maladie» maintenant retirée et intitulé de manière fallacieuse «Pour plus d'efficience et de qualité dans l'assurance-maladie» vient tenter de court-circuiter les débats en cours. Le CC et votre assemblée des délégués et vous-mêmes vous êtes massivement engagés dans la campagne qui a trouvé son issue favorable le $1^{\text {er }}$ juin dernier. Le peuple suisse, avec 69,5\% de non à ce contreprojet fallacieux, a donné une réponse qui devrait éliminer définitivement de la discussion la liberté de contracter et toute stratégie reposant sur une limitation du libre choix du médecin par le patient. Et pourtant déjà certains milieux imaginent déjà de revenir à la charge au niveau de la loi ...

J'en viens à mes conclusions. Premièrement, le CC de la législature 2004-2008 a fait des progrès absolument considérables. Fatalement il devait se chercher quelque peu après les divers épisodes confus et souvent malheureux vécus précédemment. Cette absence d'orientation claire au début a débouché sur des contestations qui n'ont pas toujours été très productives. Il n'en demeure pas moins que la communication et les contacts politiques de la FMH se sont considérablement améliorés au cours de la dernière législature. Votre président et différents membres du Comité central ont régulièrement été présents lors des ré- unions du groupe parlementaire «Santé». Des démarches multiples ont été effectuées auprès de parlementaires dans d'autres cercles, que ce soit l'USPL par exemple, d'autres associations et groupements, ou sur rendez-vous personnel. Les communiqués de la FMH ont commencé à porter et avoir une réelle influence sur les décisions des Commissions des Etats ou du National, ainsi que des plenums respectifs. Même le chef du département s'est mis à nous prendre davantage au sérieux. A titre de conseiller national, je ne me suis plus trouvé comme acteur solitaire, pris au dépourvu par des initiatives intempestives, ou dans le tourbillon de propos contradictoires. Au contraire, mes initiatives ont bénéficié d'un soutien de mieux en mieux structuré. Cela a débouché maintenant sur la mise en place d'un service de communication FMH digne de ce nom avec l'appui d'une agence spécialisée.

Deuxièmement, le succès politique de la FMH n'a de chance que si nous venons avec des propositions d'intérêt public. Nous n'avons strictement aucune chance si nous en restons à nos propres intérêts professionnels immédiats. Il s'est avéré donc primordial de situer notre profession et son rôle dans le cadre du système de santé. Nous avons centré notre action sur le principe du dialogue partenarial reconnu dans l'ensemble de l'économie, et sur la défense de la liberté thérapeutique. La tendance devient en effet de plus en plus grande de vouloir inscrire directement dans la loi la procédure de prise en charge - c'est le cas en particulier dans la loi sur les stupéfiants et dans l'ordonnance sur la transplantation. Avec les multiples législations en cours d'élaboration ou nécessitant une révision, Loi sur la procréation assistée, Loi sur l'expérimentation humaine, Loi sur la prévention, ou simplement et surtout la LAMal, etc., les tentations de prescriptions ou d'asservissement à un organe directeur ou tout simplement aux caisses sont grandes. Se retrancher derrière des principes d'intérêts généraux simples et clairs est la seule approche crédible. Nos conditions de travail font partie d'une autre négociation dont l'issue dépend des succès de principe que nous aurions par ailleurs obtenus ou non. Il est donc totalement illusoire et contreproductif de venir charger le bateau avec une multiplicité d'autres considérations certes importantes à nos yeux, mais qui ne font que créer la confusion et la suspicion quant à nos objectifs véritables.

Troisièmement, l'une des stratégies fondamentales pour obtenir ces succès dépend de l'unité dont fait preuve notre association professionnelle. Parler d'une seule voix est donc crucial. Rien n'est plus destructif que l'intervention 
parallèle de groupe d'intérêts particuliers. Nous avons maintenant procédé à des réformes de structures avec la mise sur pied d'une Assemblée des délégués qui doit faciliter la communication et permettre d'établir des relations de confiance satisfaction. Les temps ont changé. Les médecins sont maintenant un groupe d'intérêts et d'influence comme les autres. Il importe donc de se documenter d'abord de manière exhaustive et de respecter un certain nombre de règles. La pre-

\section{Les communiqués de la FMH ont commencé à porter et avoir une réelle influence sur les décisions des Commissions des Etats ou du National, ainsi que des plenums respectifs. Même le chef du département s'est mis à nous prendre davantage au sérieux}

suffisantes. Le mot «confiance» joue là un rôle absolument central. Dans le cadre de SwissDRG, différents groupes de travail ont été constitués afin de garantir un flux d'informations et d'interventions régulier des sociétés de discipline et des spécialistes de ces problèmes. Notre délégué au Conseil d'administration, le Dr P.-F. Cuénoud rapporte régulièrement à l'AD. C'est l'exemple même d'une démarche qui donne de bons résultats sur le plan interne et sur le plan externe. Il s'agit d'accorder la même confiance au président et à ses vice-présidents et d'admettre aussi qu'ils peuvent être appelés à prendre des mesures d'urgence au gré des événements qui ne peuvent que se situer dans la logique générale qui a déjà été exposée aux différentes instances compétentes.

Enfin il y a lieu d'améliorer notre culture politique à tous les niveaux. Ce n'est pas simplement parce que les médecins formulent des revendications fermes qu'ipso facto ils doivent obtenir mière est le respect de l'adversaire; la deuxième de concevoir des messages courts et simples, rapidement compréhensibles. Les parlementaires lisent difficilement plus d'une page A4. Enfin la troisième est de respecter les décisions démocratiques et nos structures institutionnelles pour parler d'une seule voix. Sans cet effort de confiance, il n'y a pas de succès possible.

La FMH s'est profondément réorganisée et démocratisée. Sa crédibilité politique a considérablement augmenté ces dernières années. Cette voie nouvelle nous permettra d'obtenir davantage de succès que par le passé. Il est à souhaiter aussi que le dialogue partenarial et l'état d'esprit constructif qui devrait l'accompagner s'améliorent. Il devrait figurer dans la loi comme principe premier de recherche de solutions. Mes meilleurs vœux vont au nouveau CC et à la FMH avec tous mes remerciements pour la confiance qui m’a été accordée tout au long de ces années. 